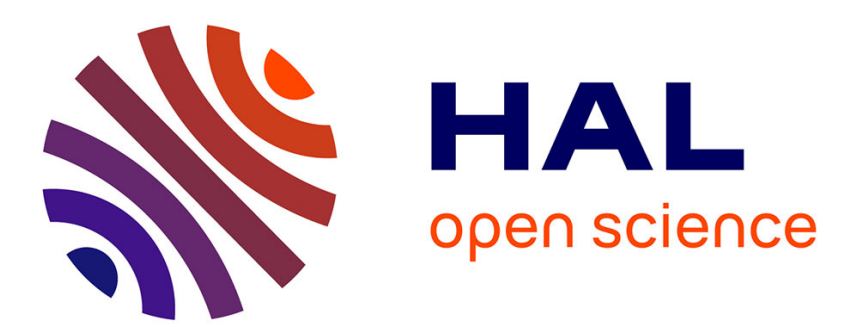

\title{
Reduced multipath channel modelling preserving representative GNSS receiver testing
}

F. Ribaud, M. Ait-Ighil, S. Rougerie, J. Lemorton, Olivier Julien, F. Pérez-Fontan

\section{- To cite this version:}

F. Ribaud, M. Ait-Ighil, S. Rougerie, J. Lemorton, Olivier Julien, et al.. Reduced multipath channel modelling preserving representative GNSS receiver testing. ION GNSS 2016, 29th International Technical Meeting of the Satellite Division of the Institute of Navigation, 5 ., Sep 2016, PORTLAND, United States. 10.33012/2016.14800 . hal-01404404

\section{HAL Id: hal-01404404 https://hal.science/hal-01404404}

Submitted on 28 Nov 2016

HAL is a multi-disciplinary open access archive for the deposit and dissemination of scientific research documents, whether they are published or not. The documents may come from teaching and research institutions in France or abroad, or from public or private research centers.
L'archive ouverte pluridisciplinaire HAL, est destinée au dépôt et à la diffusion de documents scientifiques de niveau recherche, publiés ou non, émanant des établissements d'enseignement et de recherche français ou étrangers, des laboratoires publics ou privés. 


\title{
Reduced multipath channel modelling preserving representative GNSS receiver testing
}

\author{
F. Ribaud ${ }^{1}$, M. Ait-Ighil ${ }^{1}$, S. Rougerie ${ }^{2}$, J. Lemorton ${ }^{1}$, O. Julien ${ }^{3}$, F . Pérez-Fontan ${ }^{4}$ \\ ${ }^{1}$ ONERA - The French Aerospace Lab: DEMR, Toulouse, France \\ ${ }^{2}$ CNES: RF/ITP, Toulouse, France \\ ${ }^{3}$ ENAC: Signal Processing and Telecommunications Laboratory, Toulouse, France \\ ${ }^{4}$ University of Vigo, Vigo, Spain
}

The increasing need for accuracy in GNSS positioning applications requires a thin knowledge of the impact of the urban propagation channel on the receiver's tracking loops. Therefore, new receivers' performance assessment requires the study of their behavior in a multipath environment. In that context, propagation channel synthesizers have to be able to provide multipath parameters that are representative of a real environment. Then, the receiver can be put to test by using these multipaths parameters to quantify its resulting positioning error.

In order to simulate a realistic environment, the channel synthesizer usually generates a high number of multipaths. This characteristic can prevent an optimal receiver performance evaluation, especially when using a hardware channel emulator, whose multipath production capacity does not normally exceed 10 units per satellite (even if the total number of channels can reach 36 or 72 in the most powerful emulator). Therefore, this study aims at elaborating reduction methods of the channel impulse response and comparing their capacity to preserve the receiver's positioning error induced by the multipath channel, in order to advocate the use of one of these methods to answer the multipath channel reduction problem.

\section{Elaboration of 3 reduction methods}

The first part of the study consists in the description of the considered reduction methods. They represent 3 different approaches to the problem:

\section{A. Aggregation methods}

First, the channel impulse response can be reduced by coherently summing (amplitude and phase) groups of multipaths. To do so, a clustering method has been implemented in order to create clusters of multipaths according to Delay and Doppler proximity criteria. The multipaths of the reduced channel are the centers of the clusters, located at the barycenter of the multipaths of the clusters. Such a reduction technique has been chosen for ease of application to any impulse response and its preservation of the multipaths power-delay spread and Doppler spectrum. Figure 1 illustrates an example of channel impulse response reduction using the Clustering technique, with 5 multipaths in the reduced channel. 

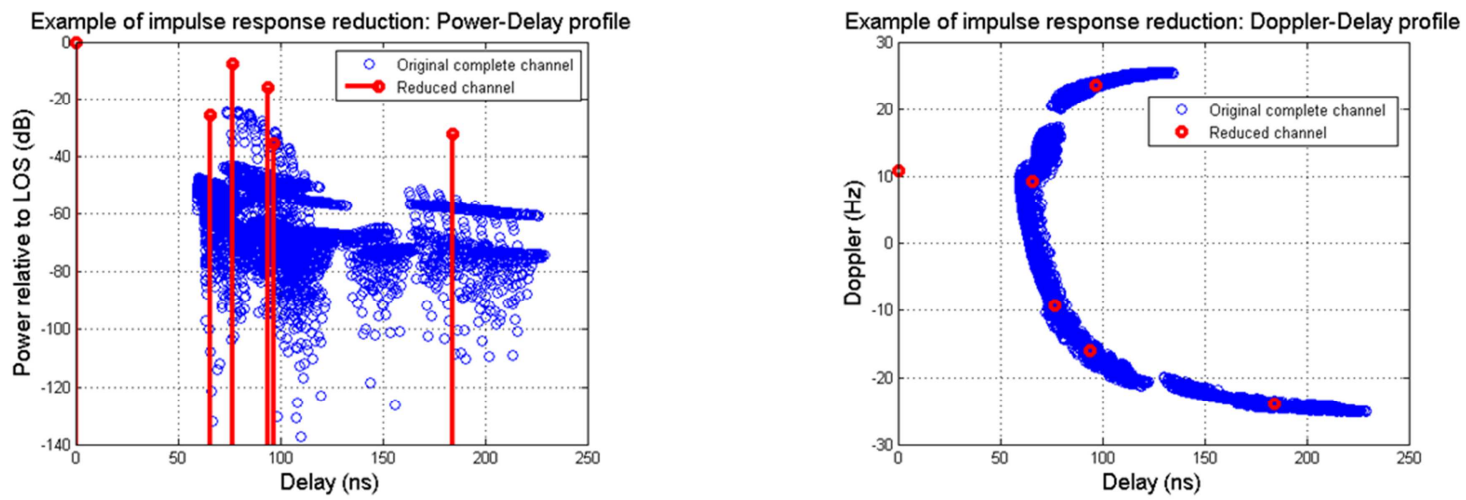

Figure 1. Example of impulse response reduction with the Clustering technique

\section{B. Application of parametric methods}

A second approach consists in optimizing the parameters of the reduced channel multipaths (delay, Doppler and amplitude) to minimize a cost function. Knowing that the receiver uses the correlation of the incoming GNSS signal with its local replica to compute the tracking loop discriminator, the cost function has been chosen to be the difference between this theoretical correlation function convoluted with the complete original multipath channel and the reduced one. The SAGE (Space Alternating Generalized Expectation maximization) iterative algorithm has been used to perform this optimization, like in [2]. The multipaths parameters output of the algorithm constitute the reduced channel impulse response. Figure 2 illustrates an example of channel impulse response reduction using the parametric technique, with 5 multipaths in the reduced channel. An example of this optimization realization is displayed on fig. 3, which represents the autocorrelation functions of the original complete multipath channel and reduced one. The Line Of Sight (LOS) contribution has not been represented to highlight the importance of the multipaths parameters.
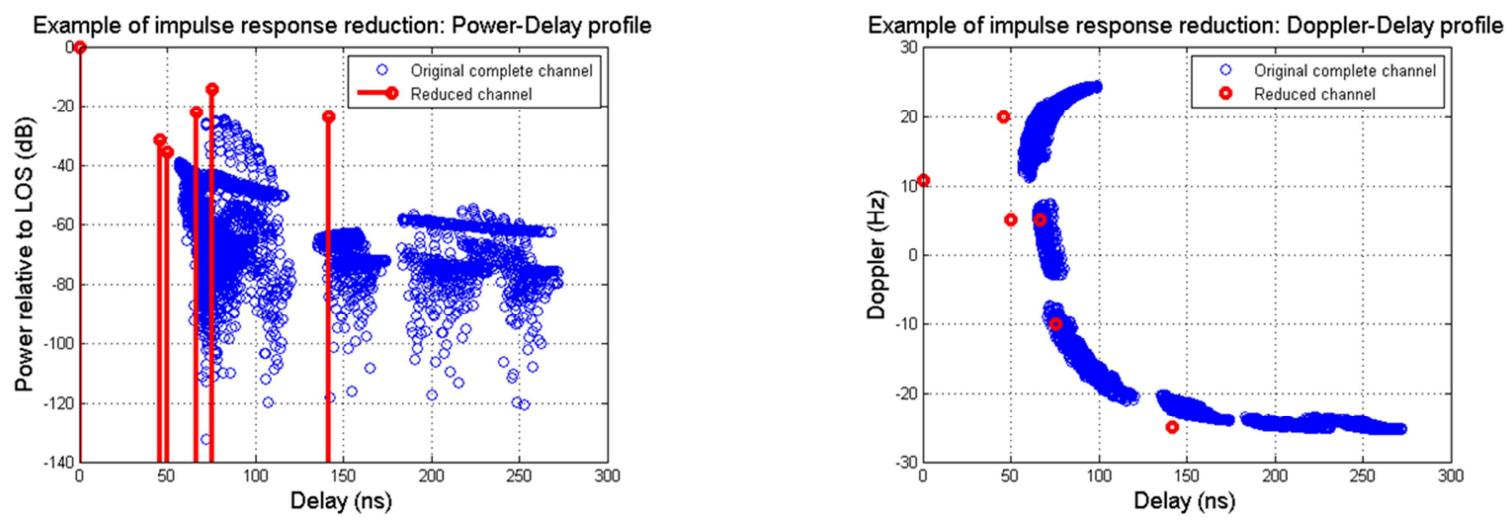

Figure 2. Example of impulse response reduction with the parametric technique 


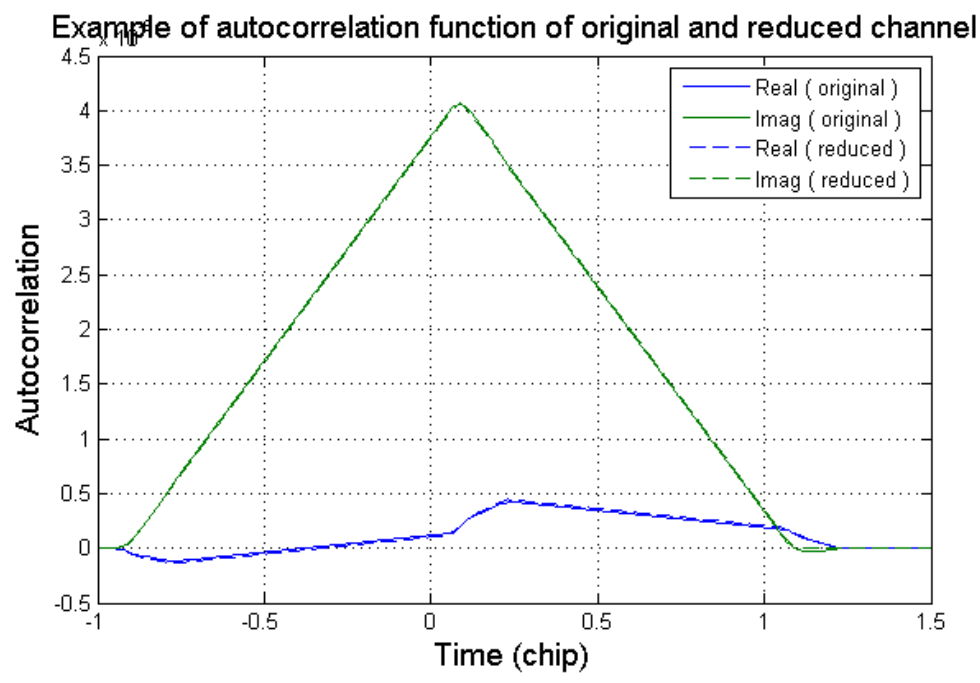

Figure 3. Example of the result of the minimization of the autocorrelation difference (without LOS)

\section{Statistical approach}

The last approach considered in this study computes the multipaths of the reduced channel as the realization of a stochastic process. The temporal evolution of the reduced channel multipaths delays and Doppler shifts follow a discrete state and time Markov channel, like in [3]. In other terms, the multipaths parameters depend at a given time on the present delay and Doppler statistic distribution and on the one at the precedent epoch to calculate the transition probabilities. The complex amplitudes of those contributions are drawn from the complete channel impulse response, knowing their delay. The multipaths of the reduced channel are realizations of this stochastic process. It can be remarked that contrary to the two previous reduction methods, this one is not deterministic and its performances can variate from realization to realization. Figure 4 illustrates an example of channel impulse response reduction using the statistical technique, with 5 multipaths in the reduced channel. Figure 5 illustrates the stochastic evolution of the delay of the multipaths of the reduced channel.
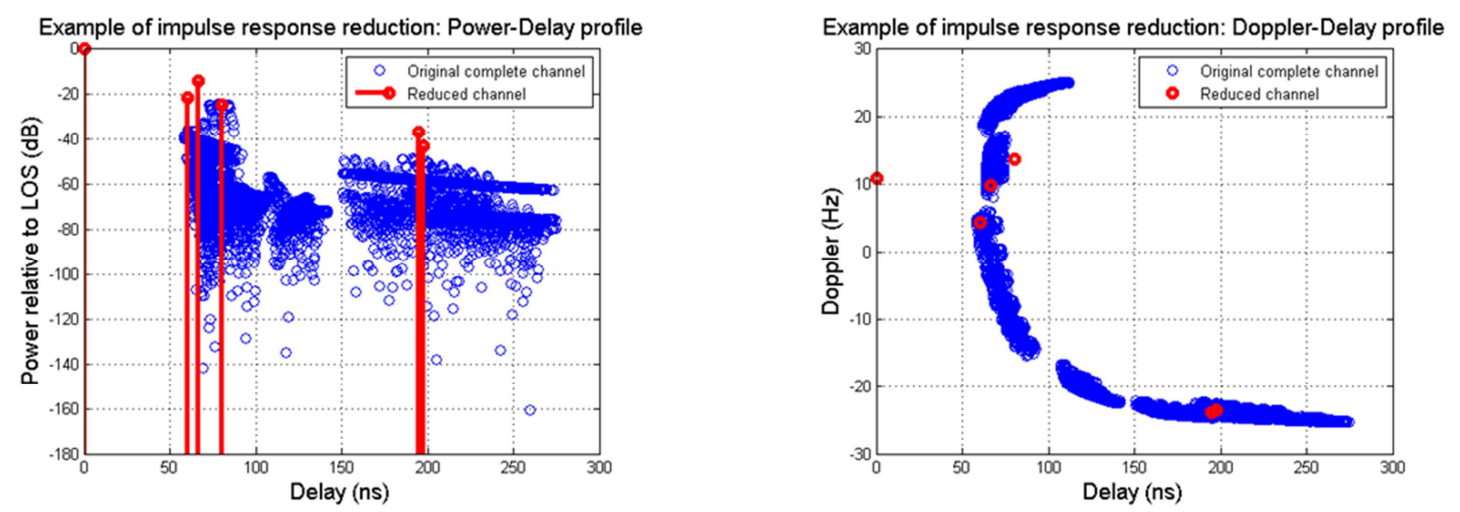

Figure 4. Example of impulse response reduction with the statistic technique 


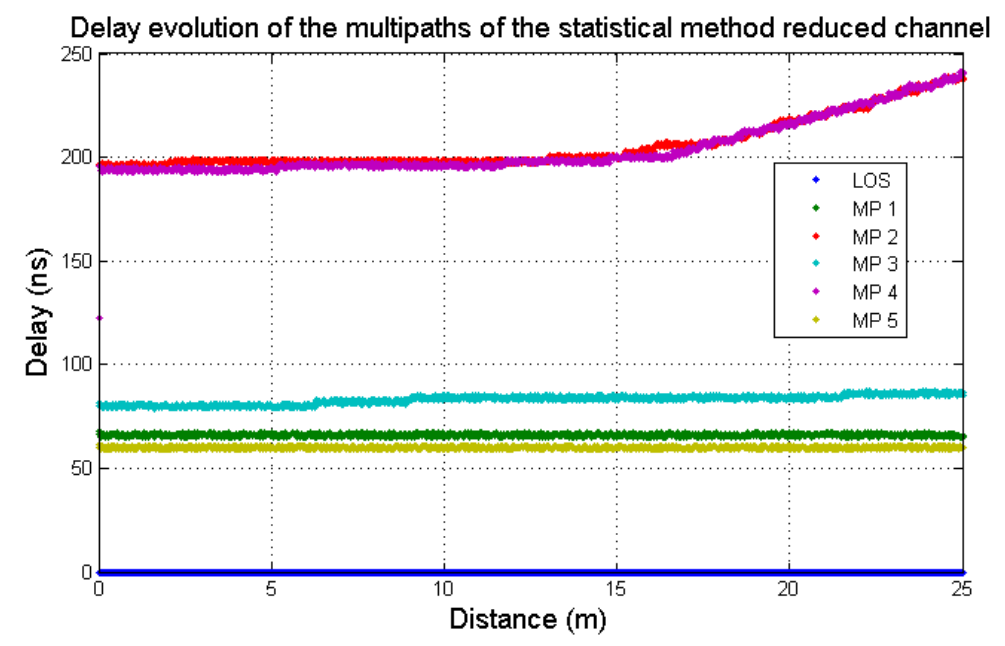

Figure 5. Delay evolution of the statistical method reduced channel multipaths along the receiver's way

\section{Study of the pseudo-range error preservation}

The SCHUN (Simplified Channel for Urban Navigation) channel synthesizer [1] has been chosen for its channel accuracy/computation time ratio to generate a reference channel impulse response in a virtual environment in which the receiver moves (multipaths delay, Doppler and complex amplitude). This reference scenario is represented on fig. 6.The receiver moves along the blue line at a speed of $5 \mathrm{~m} . \mathrm{s}^{-1}$. A single satellite emitter has been considered located at azimuth $30^{\circ}$ and elevation $35^{\circ}$. The reduction methods have been applied to this reference scenario along the receiver's trajectory to compare their pseudo-range error preservation performances. The number of multipath has been reduced by the 3 reduction methods from several thousands in the reference channel to 5 units in one case and to 10 units in a parallel study. The performance of the different multipath reduction methods can then be compared according to several criteria.

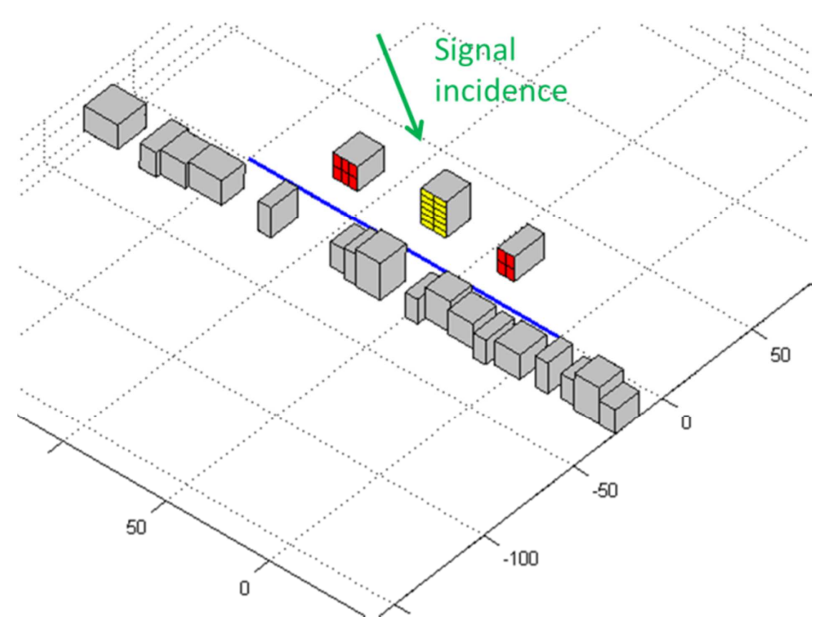

Figure 6. Reference scenario 


\section{A. Discriminator error preservation}

First, the impact of the channel reduction process has been observed through its impact on the tracking loop's discriminator output. The principle of this criterion is to compute the deviation of the discriminator due to the multipath channel considering a given discriminator, Chip Spacing (CS) and signal modulation. This study offers a first look at the multipath channel impact on the receiver by looking at its impact on the tracking loop stable lock point (instead of the actual tracking loops). The resulting has been computed for a BPSK modulated signal along the receiver's trajectory for the reference impulse response and the different reduced ones, using an Early Minus Late (EML) Discriminator with 1 chip CS.

The comparison of the difference of static pseudo-range error between the reference channel and the different reduced channels has shown that the best reduction method according to this criterion is the optimization method (B). The clustering technique (A) preserves discriminator output error almost as good as the optimization method does. Indeed the difference between the reference channel discriminator error and the clustering reduced channel is $12 \%$ higher than the difference between the reference channel and the optimization technique reduced one.

On the contrary, the statistic method (C) has the worst performances considering this criterion. Indeed the difference between the reference channel discriminator error and the statistical method reduced channel is 3 times higher than the difference between the reference channel and the optimization technique (B) reduced one. Those statements remain the same considering a reduced channel impulse response with 5 multipaths and 10 multipaths. Figure 7 represents the cumulative distribution of the difference between the discriminator error of the reduced channels and the original one along the receiver's path. Left plot corresponds to the 5-multipath reduced channels study and the right plot the 10-multipaths study.
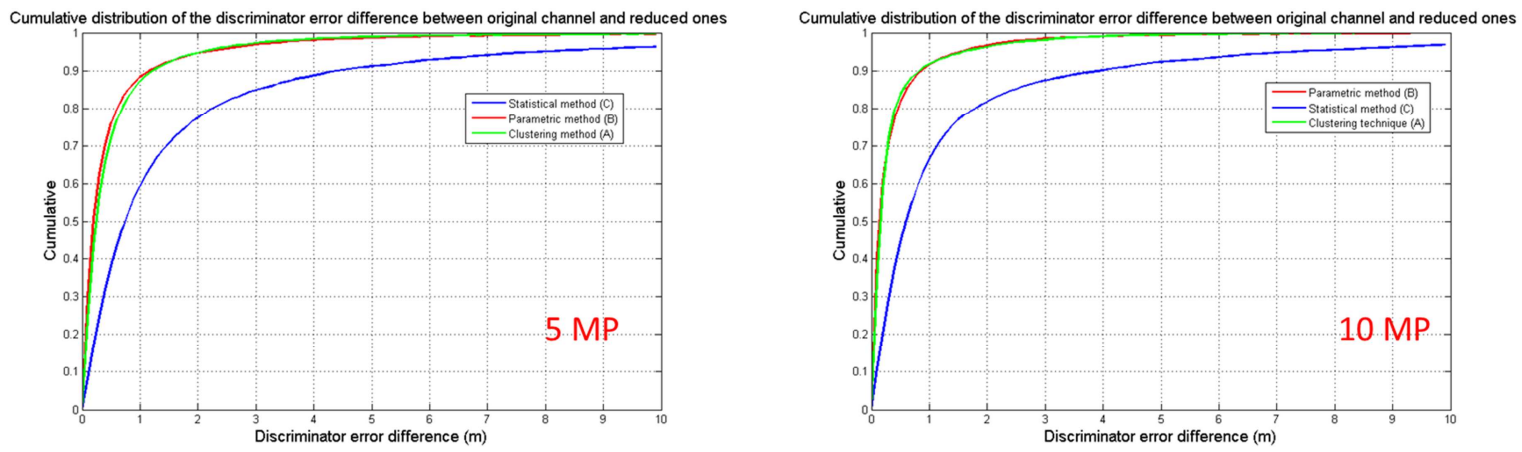

Figure 7. Cumulative distribution of the absolute difference between the discriminator error caused by the original channel and the reduced ones in case of 5 and 10 multipaths. 
B. Dynamic pseudo-range error preservation

To study the impact of the reduction method on a real receiver, a software receiver has been used to compute the receiver's pseudo-range error along its trajectory. In this section, the dynamic tracking of the GNSS signal code and carrier phase are investigated. The signal is BPSK modulated and an EML discriminator with CS=1 chip has been used for the DLL.

This subsection confirms the conclusion of the previous one concerning the hierarchy of the methods according to the preservation of their impact on the receiver: the optimization technique (B) has the best preservation performances, close to the Clustering (A) performances and far better than the statistical method (C) performances. This hierarchy among the channel reduction methods according to this static pseudo-range error criterion is the same with 5 or 10 multipaths in the reduced channels.

Moreover, it is observed that the difference between the Clustering technique and the optimization technique performances decreases with the increase of the number of multipath in the reduced channel, tending to converge. Figure 8 represents the cumulative distribution of the difference between the dynamic pseudo-range error of the reduced channels and the original one along the receiver's path. Left plot corresponds to the 5-multipath reduced channels study and the right plot the 10-multipaths study.
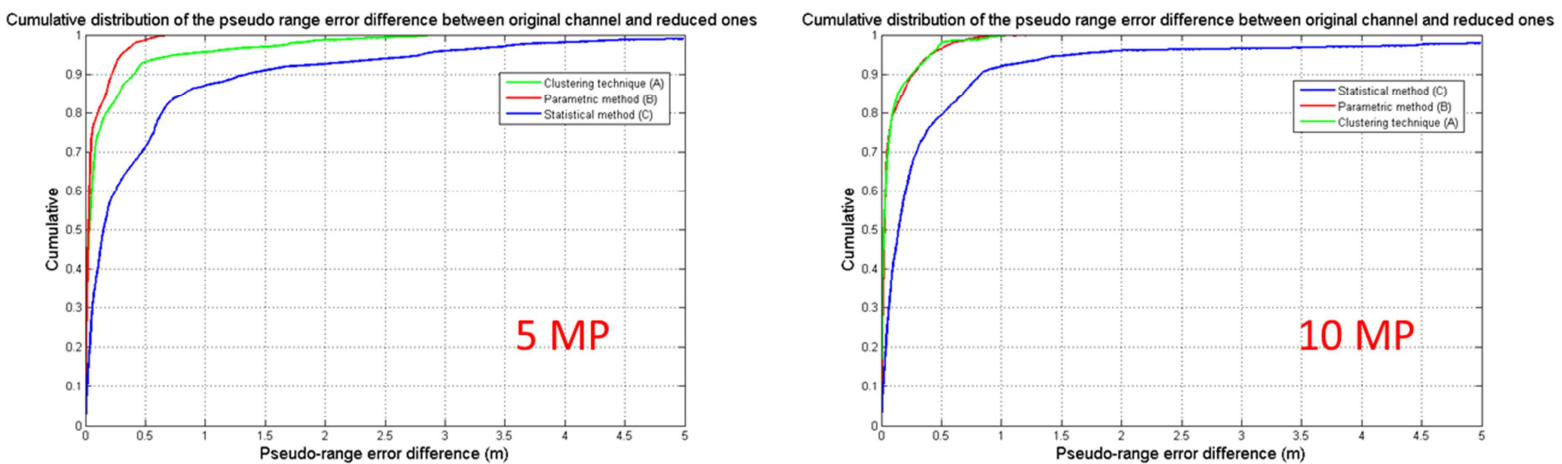

Figure 8. Cumulative distribution of the absolute difference between the discriminator error caused by the original channel and the reduced ones in case of 5 and 10 multipaths.

\section{Conclusion}

This study has presented three different reduction methods, elaborated from three different approaches (multipath aggregation, channel autocorrelation function preservation and stochastic evolution of the multipaths parameters), aiming at covering a large field of possible solutions of the channel impulse response reduction problematic. The goal of these methods is to reduce the number of multipaths from several thousands to less than 10 units impacting the receiver's positioning performances as little as possible. 
Therefore, their validity has been investigated through the preservation of the receiver's behavior. The results of this comparison show a big difference between the Clusteringparametric methods (A-B) and the statistical method (C), which corrupts the pseudo-range error much more than the two others. As a consequence, this method can be eliminated from the possible approaches to answer the multipath channel reduction problem.

The performances of the Clustering (A) and optimization (B) techniques show to be very close for 5 multipaths and show disappear in the 10 multipaths study. It is however important to mention that the parametric technique (B) has a large computation time, whereas the Clustering technique (A) computation time is negligible as compared to the receiver's multipath processing time. Therefore, the use of the Clustering technique (A) to reduce the multipath channel can be recommended for most of the applications.

Finally, this study has used both static discriminator error and dynamic receiver's tracking error to assess the performance of the reduction methods. Both show the same conclusions. As a conclusion, the discriminator error, easy to compute, could be sufficient to evaluate the performances of a given reduction method in a given scenario. This tool can be a complement to the reduction method in itself, quantifying its error on the receiver's pseudo-range error preservation, playing the role of a representativeness index.

\section{Reference:}

[1] M. Ait-Ighil "Enhanced Physical-Statistical Simulator of the Land Mobile Satellite for Multipath Modelling Applied to a Satellite Navigation System" University of Toulouse PhD Thesis, 2013.

[2] S.Rougerie, "Algorithmes de diversité d'antennes appliqués à la reception des signaux GNSS en environnement urbain et sur terminal mobile", University of Toulouse PhD Thesis, 2012.

[3] F. M. Schubert, M. Heimerl, J. Wendel "Components Count Decrease of Channel Responses Using Markov Process", $7^{\text {th }}$ ESA Workshop on Satellite Navigation Technologies, Navitech 2014. 